\title{
SPITZER OBSERVATIONS OF HIGH-REDSHIFT QSOs
}

\author{
Dean C. Hines, ${ }^{1}$ O. Krause, ${ }^{2}$ G. H. Rieke, ${ }^{2}$ X. Fan, ${ }^{2}$ M. Blaylock, ${ }^{2}$ and G. Neugebauer ${ }^{2}$ \\ Received 2006 January 19; accepted 2006 March 10; published 2006 April 5
}

\begin{abstract}
We have observed $13 z \geq 4.5$ QSOs using the Multiband Imaging Photometer for Spitzer, nine of which were also observed with the Infrared Array Camera. The observations probe rest wavelengths $\sim 0.6-4.3 \mu \mathrm{m}$, bracketing the local minimum in QSO spectral energy distributions (SEDs) between strong optical emission associated directly with accretion processes and thermal emission from hot dust heated by the central engine. The new Spitzer photometry combined with existing measurements at other wavelengths shows that the SEDs of highredshift QSOs $(z \geq 4.5)$ do not differ significantly from typical QSOs of similar luminosity at lower redshifts $(z \lesssim 2)$. This behavior supports other indications that all the emission components and physical structures that characterize QSO activity can be established by $z=6.4$. The similarity also suggests that some QSOs at high redshift will be very difficult to identify because they are viewed along dust-obscured sight lines.
\end{abstract}

Subject headings: infrared: galaxies — quasars: general — galaxies: high-redshift

\section{INTRODUCTION}

Recent optical surveys have revealed many QSOs with redshifts $z>4.5$, including several with $z>6$. High-resolution imaging indicates that their luminosities are intrinsic and not caused by lensing (e.g., Fan et al. 2001, 2003; Richards et al. 2004). X-ray (Brandt et al. 2002; Mathur et al. 2002; Shemmer et al. 2005), UV/optical, and near-IR spectroscopic observations (Fan et al. 2003; Barth et al. 2003; Maiolino et al. 2003) show that these sources share many properties with luminous QSOs at lower redshift $(z \lesssim 2)$. In particular, ultraviolet continua, high-ionization emission lines, and strong low-ionization $\mathrm{Mg}$ II and $\mathrm{Fe}$ II emission are all present in proportions consistent with the low-redshift counterparts.

Submillimeter and centimeter radio observations indicate that these QSOs have copious dust (e.g., Omont et al. 1996; Bertoldi et al. 2003a, 2003b; Robson et al. 2004; Carilli et al. 2004), but this cool material may simply be heated by massive star formation in highly luminous host galaxies and thus may be unrelated to the QSO activity. Active nuclei can heat dust directly to produce stronger mid-infrared emission than is characteristic of star formation. This behavior is common in radio galaxies and QSOs at low redshift (Neugebauer et al. 1986; Low et al. 1988, 1989; Polletta et al. 2000; Wilkes et al. 2000; Kuraszkiewicz et al. 2003; Haas et al. 1998, 2000, 2003; Shi et al. 2005). However, the Infrared Astronomical Satellite and Infrared Space Observatory were not sensitive enough to probe this type of emission in high-redshift QSOs.

The Spitzer Space Telescope (Werner et al. 2004) provides sufficient sensitivity to search for this mid-IR emission from even the most highly redshifted QSOs currently known. As part of a large survey of luminous active galactic nuclei (AGNs), we have observed 13 high-redshift $(z>4.5)$, UV/optically selected QSOs with the goal of comparing their spectral energy distributions (SEDs) with lower redshift objects. In this Letter, we use the new observations to study the rest wavelengths between 0.6 and $4.3 \mu \mathrm{m}$, a region containing the characteristic local minimum $(\lambda \sim 1.5 \mu \mathrm{m})$ in QSO SEDs as well as the thermal emission from the hot $\left(T_{\text {dust }} \geq 300 \mathrm{~K}\right)$, AGNheated dust. A complementary General Observer program will

\footnotetext{
${ }^{1}$ Space Science Institute, 4750 Walnut Street, Suite 205 Boulder, CO 80301. ${ }^{2}$ Steward Observatory, The University of Arizona, 933 North Cherry Avenue, Tucson, AZ 85721.
}

report on a larger sample of high-redshift QSOs and will investigate their SEDs from X-rays through the Spitzer and radio wavelengths (X. Fan et al. 2006, in preparation).

\section{OBSERVATIONS}

Images at $24 \mu \mathrm{m}\left(5^{\prime \prime} .25\right.$ pixel $^{-1} \times 5.25$ pixel $\left.^{-1}\right)$ were obtained for each object using the photometry mode of the Multiband Imaging Photometer for Spitzer (MIPS; Rieke et al. 2004) as part of the MIPS Guaranteed Time Observation program titled "The Far-IR Spectral Energy Distributions of Luminous Active Galactic Nuclei" (PID 82). After initial processing by the Spitzer Science Center (SSC) pipeline (S10.5.0) to provide reconstructed pointing information, the MIPS data were further processed into a final image using the MIPS Data Analysis Tool (DAT, ver. 2.71) developed by the MIPS Instrument Team (Gordon et al. 2004, 2005). Aperture photometry was performed on the images with IDP3 (ver. 2.9; Schneider \& Stobie 2002), using a 14."7 target aperture and a background annulus from 29".4 to 41 1.7. The median background per pixel was scaled to the appropriate target aperture size and subtracted from the summed target aperture flux for each object. The final flux in instrumental units was then corrected to an infinite aperture, and conversion to physical flux density units was performed by multiplying the instrumental total flux by the MIPS calibration factor (MIPS Data Handbook, ver. 3.1). Random uncertainties in the background-subtracted estimates of on-source flux were estimated from the rms pixel-to-pixel dispersion inside the background annulus measured on the image and scaled to the area of the target aperture. The final uncertainties are dominated by the absolute calibration uncertainty of $10 \%$.

Infrared Array Camera (IRAC; Fazio et al. 2004) observations of nine QSOs were obtained in channels 1, 2, 3, and 4 (3.6, 4.5, 5.8, and $8.0 \mu \mathrm{m})$. Images were obtained as Basic Calibrated Data products from the SSC data pipeline (S11.5.0) as described in the SOM 4.6 and the Pipeline Description Document available through the SSC. Aperture photometry was performed using a 3 pixel (3".66) target aperture and background annuli from 3-7 pixels. Other aspects of the photometry were similar to the procedures used at $24 \mu \mathrm{m}$. The measurement uncertainties were estimated from the rms noise in the background annulus. The uncertainty in absolute calibration is 3\%$5 \%$ (Reach et al. 2005).

The measured flux densities for each object are presented in 
TABLE 1

Spitzer Observed Photometry of High-Redshift QSOs

\begin{tabular}{|c|c|c|c|c|c|c|c|c|c|c|c|}
\hline \multirow[b]{2}{*}{ ОвЈеСт } & \multirow[b]{2}{*}{$z_{\mathrm{em}}$} & \multicolumn{10}{|c|}{ Flux Density $^{\mathrm{a}}(\mathrm{mJy})$} \\
\hline & & $3.6 \mu \mathrm{m}^{\mathrm{b}}$ & $\sigma(3.6 \mu \mathrm{m})^{\mathrm{c}}$ & $4.5 \mu \mathrm{m}^{\mathrm{b}}$ & $\sigma(4.5 \mu \mathrm{m})^{\mathrm{c}}$ & $5.6 \mu \mathrm{m}^{\mathrm{b}}$ & $\sigma(5.8 \mu \mathrm{m})^{\mathrm{c}}$ & $8.0 \mu \mathrm{m}^{\mathrm{b}}$ & $\sigma(8.0 \mu \mathrm{m})^{\mathrm{c}}$ & $24 \mu \mathrm{m}^{\mathrm{b}}$ & $\sigma(24 \mu \mathrm{m})$ \\
\hline BR J0006-6208 .......... & $4.51^{\mathrm{d}}$ & 0.316 & 0.001 & 0.256 & 0.002 & 0.345 & 0.012 & 0.605 & 0.013 & 3.34 & 0.13 \\
\hline BR J0324-2918 & 4.63 & 0.375 & 0.001 & 0.276 & 0.002 & 0.296 & 0.009 & 0.301 & 0.011 & 1.47 & 0.14 \\
\hline SDSS J0836+0054 & 5.82 & & & & $\ldots$ & $\ldots$ & & & & 1.01 & 0.10 \\
\hline SDSS J0940+5848 & 4.665 & 0.232 & 0.001 & 0.164 & 0.001 & 0.152 & 0.006 & 0.240 & 0.006 & 1.49 & 0.06 \\
\hline SDSS J0941+5947 & 4.82 & 0.128 & 0.001 & 0.100 & 0.001 & 0.0981 & 0.006 & 0.160 & 0.005 & 0.60 & 0.05 \\
\hline SDSS J1048+4637 _.... & 6.23 & $\ldots$ & $\ldots$ & $\ldots$ & $\ldots$ & $\ldots$ & $\ldots$ & $\ldots$ & & 0.86 & 0.09 \\
\hline PSS J1140+6205 $\ldots \ldots \ldots$ & 4.51 & 0.224 & 0.001 & 0.151 & 0.002 & 0.202 & 0.11 & 0.249 & 0.009 & 1.73 & 0.15 \\
\hline SDSS J1148+5251 $\ldots \ldots$ & 6.41 & $\ldots$ & $\ldots$ & $\ldots$ & $\ldots$ & $\ldots$ & $\ldots$ & $\ldots$ & $\ldots$ & 1.52 & 0.12 \\
\hline BR $1202-0725 \ldots \ldots \ldots$ & 4.694 & 0.461 & 0.001 & 0.375 & 0.001 & 0.410 & 0.001 & 0.657 & 0.002 & 2.37 & 0.24 \\
\hline PC $1247+3406 \ldots \ldots \ldots$ & 4.897 & 0.142 & 0.001 & 0.118 & 0.001 & 0.110 & 0.006 & 0.168 & 0.006 & 0.81 & 0.14 \\
\hline PSS J1715+3809 _....... & 4.52 & 0.222 & 0.001 & 0.175 & 0.002 & 0.230 & 0.010 & 0.373 & 0.010 & 2.06 & 0.07 \\
\hline PSS J1723+2243 $\ldots \ldots \ldots$ & 4.52 & 1.197 & 0.003 & 0.969 & 0.003 & 1.062 & 0.010 & 1.286 & 0.010 & 6.21 & 0.15 \\
\hline BR 2237-0607. & 4.558 & $\ldots$ & $\ldots$ & $\ldots$ & $\ldots$ & $\ldots$ & $\ldots$ & $\ldots$ & $\ldots$ & 3.49 & 0.09 \\
\hline
\end{tabular}

${ }^{\text {a }}$ The filters are sufficiently narrow that uncertainties due to assumed SEDs and the color corrections are $<5 \%$ (IRAC and MIPS Data Handbooks). The conversions to millijanskys given in SOM 4.6 are assumed here.

${ }^{\mathrm{b}}$ Wavelengths are the band designations for each instrument. The wavelengths corresponding to the monochromatic flux density are listed in the SOM 4.6. Bandpasses are $21 \%, 23 \%, 25 \%$, and 36\% for IRAC bands 3.6, 4.5, 5.8, and $8.0 \mu \mathrm{m}$ and 20\%, 27\% and 22\% for MIPS 24,70 , and $160 \mu \mathrm{m}$.

${ }^{\mathrm{c}}$ Measurement uncertainties only. Absolute calibration uncertainties are 3\%-5\% for IRAC (Reach et al. 2005) and 10\% for MIPS $24 \mu \mathrm{m}$ (SOM 4.6).

${ }^{\mathrm{d}}$ For our object selection and analysis, we adopt the discovery redshift from Storrie-Lombardi et al. (2001). A slightly lower redshift $(z=4.445)$ is listed in Péroux et al. (2001).

Table 1 along with their measurement uncertainties. The QSOs were detected with high signal-to-noise ratio in the MIPS $24 \mu \mathrm{m}$ band and in all four bands for the nine objects that were observed with IRAC. The objects were unresolved, except for BR $1202-0725$, which is known to have a nearby, IR-bright companion galaxy 2".6 to the northwest (e.g., Hu et al. 1996; Ohyama et al. 2004). For this object, the photometry in the IRAC and MIPS bands was rederived using a two-component point-spread function fit. Only the flux densities attributable to the QSO are presented in Table 1.

\section{SPECTRAL ENERGY DISTRIBUTIONS}

Figure 1 shows the rest-frame UV through mid-IR SEDs of the high-redshift sample. A composite SED for radio-quiet QSOs is also shown in each panel for comparison (Elvis et al. 1994). The composite represents the average behavior of lowredshift QSOs, but individual objects can show significant deviations. In Figure 1 $c$, we also present the radio-loud composite SED from Elvis et al. (1994) and a composite based on the Palomar-Green (PG) QSOs (Sanders et al. 1989). The highredshift QSOs generally match the composite SEDs and would not be obviously distinguishable from typical, optically selected luminous QSOs at low redshift. In particular, the rise in the high-redshift SEDs for $\lambda \gtrsim 1.5 \mu \mathrm{m}$ is likely to be the signature of thermal emission from hot dust (e.g., Rieke \& Lebofsky 1981; Cutri et al. 1985; Barvainis 1990; Elvis et al. 1994; Haas et al. 1998, 2000, 2003).

The deviations from the composite SEDs are not surprising. A few objects (e.g., SDSS J1148+5251, BR 0004-6224) show a more rapid rise into the infrared, and SDSS J0836+0054 is radio-loud (Petric et al. 2003), but their behavior still lies within the scatter seen for lower redshift objects (Elvis et al. 1994). The rapid decrease for $\lambda \leq 0.2 \mu \mathrm{m}$ in the SEDs of the three QSOs with $z \geq 5.8$ is caused by absorption from the Ly $\alpha$ forest in the observed frame. In addition, the photometric bands that contain the $\mathrm{H} \alpha$ emission line in each object are elevated above the extrapolated continuum.

At low redshift, the strength of the $\lambda \approx 3.7 \mu \mathrm{m}$ infrared band is correlated with the $B$-band flux density, implying that IR emission arises from hot dust heated directly by the UV/optical continuum from the central active nucleus (Rieke \& Lebofsky 1981; Cutri et al. 1985; Barvainis 1990; Haas et al. 2003). The rest-frame flux density ratios $F_{\nu}(3.7 \mu \mathrm{m}) / F_{\nu}(B)$ derived from the three composite SEDs shown in Figure $1 c$ are 7.8, 6.0, and 5.6 for the radio-quiet, radio-loud, and PG QSOs, respectively. A recent Spitzer investigation of the SEDs of unreddened, lowredshift QSOs identified in the Sloan Digital Sky Survey (SDSS) suggests that this ratio can be somewhat larger $(\sim 8-$ 9; Richards et al. 2006). The ratio for our sample of highredshift QSOs is $8.1 \pm 1$. X-ray-to-UV/optical emission is also correlated, with the X-ray-to-UV/optical spectral index depending on the luminosity of the AGN, but there is a negligible dependence of the spectral index on redshift (e.g., Strateva et al. 2005). These correlations together suggest that the source of the $3.7 \mu \mathrm{m}$ emission in QSOs is the same over a range of luminosities and for redshifts from $z \sim 0.1$ to 6.4 , and that the mid-infrared feature is emitted from dust heated directly by the central active nucleus.

\section{DISCUSSION}

Many lines of evidence suggest that the high-redshift QSOs are essentially indistinguishable from their lower redshift counterparts. The rest-frame UV/optical continua and emission lines (e.g., Barth et al. 2003; Fan et al. 2004), and the derived metallicities, are all typical of low-redshift QSOs (e.g., Pentericci et al. 2002; Maiolino et al. 2003). The objects also have similar X-ray (Brandt et al. 2002; Mathur et al. 2002), submillimeter (Bertoldi et al. 2003a; Robson et al. 2004), and radio properties (Bertoldi et al. 2003b; Carilli et al. 2004; Frey et al. 2005). Estimates of the masses of the supermassive black holes in some of these high-redshift systems imply $M_{\mathrm{SMBH}} \sim 10^{9} M_{\odot}$ (e.g., Willott et al. 2003), comparable to low-redshift QSOs. Our Spitzer measurements strengthen the evidence that QSOs have similar properties over the entire range of observed redshifts. Thus, the fundamental structures that characterize luminous QSOs in the local universe, including hot dust heated directly by the active nucleus, were already in place in these objects $\sim 900 \mathrm{Myr}$ after the big bang. ${ }^{3}$

$$
{ }^{3} H_{0}=75 \mathrm{~km} \mathrm{~s}^{-1} \mathrm{Mpc}^{-1}, \Omega_{M}=0.3 \text {, and } \Omega_{\Lambda}=0.7 .
$$




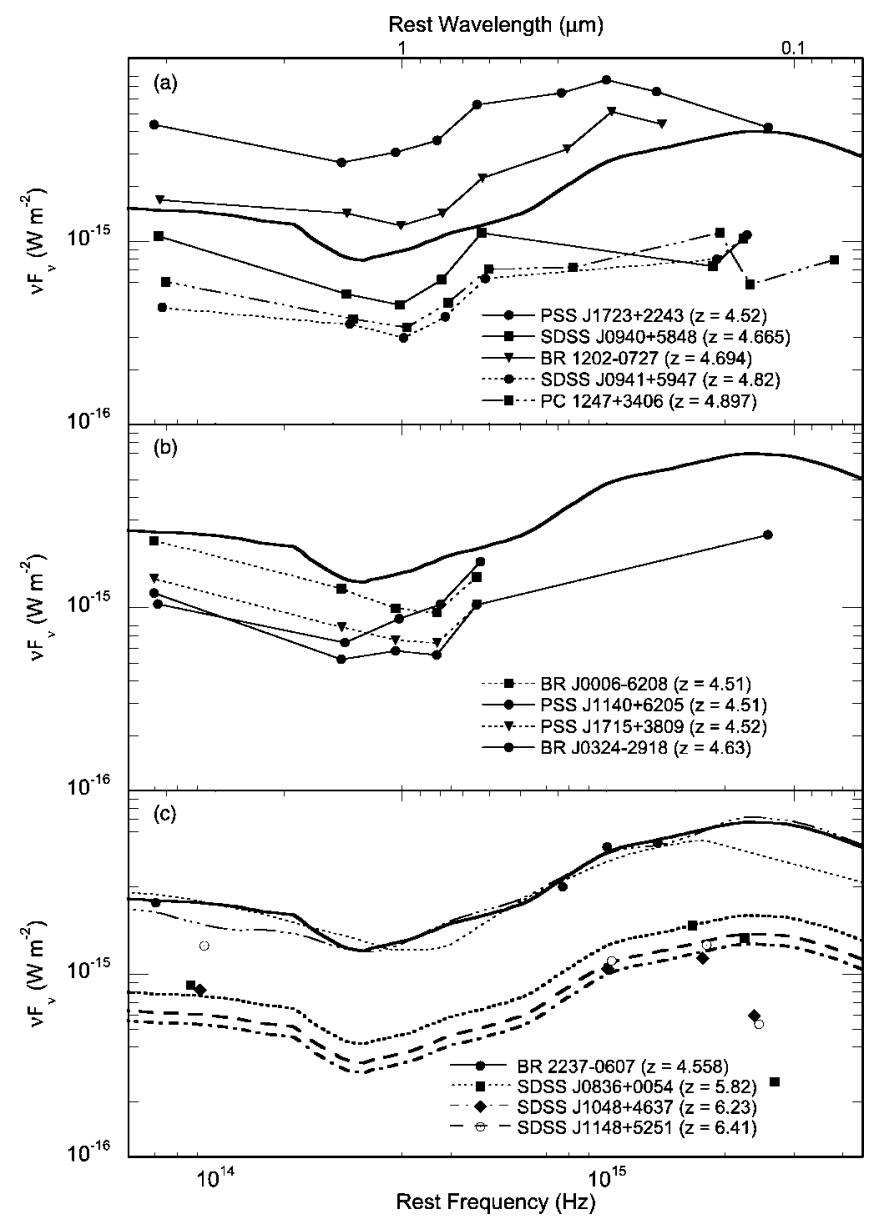

FIG. 1.-Rest-frame SEDs of high-redshift QSOs observed by Spitzer. Ground-based optical and near-IR data are also included for most objects. The objects and redshifts are indicated in the legends. Panels $a$ and $b$ show objects for which both MIPS and IRAC observations were obtained. The heavy solid curves (without individual data points) in panels $a$ and $b$ show the Elvis et al. (1994) radio-quiet QSO composite SED for comparison. Panel $c$ shows objects for which only MIPS $24 \mu \mathrm{m}$ observations were obtained. Panel $c$ also presents the Elvis et al. (1994) radio-quiet composite SED, but in this panel the SED is fitted to the rest-frame UV/optical photometry for each object. For comparison, BR 2237-0607 is also fit with the radio-loud composite SED of Elvis et al. (1994; dotted line) and a composite SED constructed from PG QSOs (Sanders et al. 1989; triple-dot, long-dashed line).

The presence of substantial amounts of both cold and hot dust around the high-redshift QSOs (e.g., Bertoldi et al. 2003a; Robson et al. 2004; this work) is consistent with their apparently unobscured ultraviolet continua only if, as with lower redshift QSOs (e.g., Neugebauer et al. 1986; Low et al. 1988, 1989), the dust is in structures that leave some lines of sight unobscured. Many of the ultra- and hyperluminous infrared galaxies have been shown to harbor QSOs that are typical of UV/optically selected objects but are obscured from direct view by dust (e.g., Hines et al. 1995, 1999; Young et al. 1996; Goodrich et al. 1996; Tran et al. 2000). The many so-called type 2 QSOs that have been identified (at $z \leq 1$ ) in the SDSS (Zakamska et al. 2003, 2004, 2005) and with Spitzer (e.g., Martínez-Sansigre et al. 2005) also must have obscuring material. Possibilities for these structures include the circumnuclear torus envisioned in simple unified schemes (e.g., Antonucci 1993; Rowan-Robinson 1995; Urry \& Padovani 2000) or more complex structures in disk winds (e.g., Konigl \& Kartje 1994; Kartje \& Konigl 1996; Elvis 2000).

In this context, the currently identified high-redshift QSOs would then represent only a fraction of the true space density of such objects, with the remainder obscured in our direction by their circumnuclear material. Furthermore, reionization of the intergalactic medium by these objects would not be distributed spherically around each QSO but would instead extend in reionization (bi)cones analogous to the ionization bicones seen in the interstellar media of nearby QSOs.

It appears that the solid-state emission features associated with aromatic molecules play an important role in the Spitzer $24 \mu \mathrm{m}$ detections of objects out to $z \sim 2.2$ (e.g., Caputi et al. 2006). At larger redshifts, the effects of these features on the detection rates will decrease because (1) they begin to move out of the MIPS band; (2) at the extremely high galaxy luminosities required for detections, the equivalent widths of the features are reduced; and (3) the effects of low metallicity and hard UV continua will tend to destroy the carriers (e.g., Engelbracht et al. 2005 and references therein). Our results show that the rest-frame near- and mid-infrared continua of luminous AGNs remain strong to at least $z \sim 6$. Thus, we expect that such AGNs will play an increasing role in the $24 \mu \mathrm{m}$ detections above $z=2.2$ and will probably dominate the detections for $z>3$.

\section{CONCLUSION}

From new 3.5, 4.5, 5.8, 8.0, and $24 \mu \mathrm{m}$ Spitzer measurements of high- $z$ QSOs $(z \geq 4.5)$ we find that:

1. The QSOs are easily detected with IRAC and MIPS at $0.6-4.3 \mu \mathrm{m}$ in the rest frame.

2. There is no obvious evolutionary trend in the UV through mid-IR SEDs compared with low-redshift QSOs $(z \lesssim 2)$.

3. The high-redshift QSOs conform to the relationship between $L_{B}$ and $L_{3.7 \mu \mathrm{m}}$ as established for low-redshift AGNs, supporting the conclusion that the rest-frame emission near $4 \mu \mathrm{m}$ in the high-redshift objects originates from hot dust heated directly by the central active nucleus.

4. AGNs will play an increasing role in the Spitzer $24 \mu \mathrm{m}$ detections above $z=2.2$ and will probably dominate the detections for $z>3$.

Our results add further evidence that the fundamental structures of QSOs can be established by $z=6.4$, corresponding to $~ 900$ Myr after the big bang. They also suggest that there is a population of QSOs at these redshifts that will be extremely difficult to identify due to dust obscuration along the line of sight.

We thank D. Sanders and B. Wilkes for supplying electronic versions of the composite QSO SEDs from Sanders et al. (1989) and Elvis et al. (1994). We also thank M. Brotherton and B. Wills for comments that contributed significantly to the clarity of the manuscript. This publication makes use of data products from the Two Micron All Sky Survey, which is a joint project of the University of Massachusetts and the Infrared Processing and Analysis Center/California Institute of Technology, funded by the National Aeronautics and Space Administration and the National Science Foundation. We have used the NED and SIMBAD databases. This work is based (in part) on observations made with the Spitzer Space Telescope, which is operated by the Jet Propulsion Laboratory, California Institute of Technology, under NASA contract 1407. Support was provided by NASA through contract 1255094 issued by JPL/Caltech. 


\section{REFERENCES}

Antonucci, R. 1993, ARA\&A, 31, 473

Barth, A. J., Martini, P., Nelson, C. H., \& Ho, L. C. 2003, ApJ, 594, L95

Barvainis, R. 1990, ApJ, 353, 419

Bertoldi, F., Carilli, C. L., Cox, P., Fan, X., Strauss, M. A., Beelen, A., Omont, A., \& Zylka, R. 2003a, A\&A, 406, L55

Bertoldi, F., et al. 2003b, A\&A, 409, L47

Brandt, W. N., et al. 2002, ApJ, 569, L5

Caputi, K. I., et al. 2006, ApJ, 637, 727

Carilli, C. L., et al. 2004, AJ, 128, 997

Cutri, R. M., Wisniewski, W. Z., Rieke, G. H., \& Lebofsky, M. J. 1985, ApJ, 296, 423

Elvis, M. 2000, ApJ, 545, 63

Elvis, M., et al.. 1994, ApJS, 95, 1

Engelbracht, C. W., Gordon, K. D., Rieke, G. H., Werner, M. W., Dale, D. A., \& Latter, W. B. 2005, ApJ, 628, L29

Fan, X., et al. 2001, AJ, 122, 2833

$$
\text { . 2003, AJ, 125, } 1649
$$

- 2004, AJ, 128, 515

Fazio, G. G., et al. 2004, ApJS, 154, 10

Frey, S., Paragi, Z., Mosoni, L., \& Gurvits, L. I. 2005, A\&A, 436, L13

Goodrich, R. W., Miller, J. S., Martel, A., Cohen, M. H., Tran, H. D., Ogle, P. M., \& Vermeulen, R. D. 1996, ApJ, 456, L9

Gordon, K. D., et al. 2004, Proc. SPIE, 5487, 177 2005, PASP, 117, 503

Haas, M., Chini, R., Meisenheimer, K., Stickel, M., Lemke, D., Klaas, U., \& Kreysa, E. 1998, ApJ, 503, L109

Haas, M., Müller, S. A. H., Chini, R., Meisenheimer, K., Klaas, U., Lemke, D., Kreysa, E., \& Camenzind, M. 2000, A\&A, 354, 453

Haas, M., et al. 2003, A\&A, 402, 87

Hines, D. C., Schmidt, G. D., Smith, P. S., Cutri, R. M., \& Low, F. J. 1995, ApJ, 450, L1

Hines, D. C., Schmidt, G. D., Wills, B. J., Smith, P. S., \& Sowinski, L. G. 1999, ApJ, 512, 145

Hu, E. M., McMahon, R. G., \& Egami, E. 1996, ApJ, 459, L53

Kartje, J. F., \& Konigl, A. 1996, Vistas Astron., 40, 133

Konigl, A., \& Kartje, J. F. 1994, ApJ, 434, 446

Kuraszkiewicz, J. K., et al. 2003, ApJ, 590, 128

Low, F. J., Cutri, R. M., Huchra, J. P., \& Kleinmann, S. G. 1988, ApJ, 327, L41

Low, F. J., Cutri, R. M., Kleinmann, S. G., \& Huchra, J. P. 1989, ApJ, 340, L1

Maiolino, R., Juarez, Y., Mujica, R., Nagar, N. M., \& Oliva, E. 2003, ApJ, 596, L155

Martínez-Sansigre, A., Rawlings, S., Lacy, M., Fadda, D., Marleau, F. R., Simpson, C., Willott, C. J., \& Jarvis, M. J. 2005, Nature, 436, 666

Mathur, S., Wilkes, B. J., \& Ghosh, H. 2002, ApJ, 570, L5
Neugebauer, G., Miley, G. K., Soifer, B. T., \& Clegg, P. E. 1986, ApJ, 308, 815

Ohyama, Y., Taniguchi, Y., \& Shioya, Y. 2004, AJ, 128, 2704

Omont, A., McMahon, R. G., Cox, P., Kreysa, E., Bergeron, J., Pajot, F., \& Storrie-Lombardi, L. J. 1996, A\&A, 315,

Pentericci, L., et al. 2002, AJ, 123, 2151

Péroux, C., Storrie-Lombardi, L. J., McMahon, R. G., Irwin, M., \& Hook, I. M. 2001, AJ, 121, 1799

Petric, A. O., Carilli, C. L., Bertoldi, F., Fan, X., Cox, P., Strauss, M. A., Omont, A., \& Schneider, D. P. 2003, AJ, 126, 15

Polletta, M., Courvoisier, T. J.-L., Hooper, E. J., \& Wilkes, B. J. 2000, A\&A, 362,75

Reach, W. T., et al. 2005, PASP, 117, 978

Richards, G. T., et al. 2004, ApJS, 155, 257 2006, ApJ, submitted

Rieke, G. H., \& Lebofsky, M. J. 1981, ApJ, 250, 87

Rieke, G. H., et al. 2004, ApJS, 154, 25

Robson, I., Pridden, R. S., Isaak, K. G., \& McMahon, R. G. 2004, MNRAS, 351, L29

Rowan-Robinson, M. 1995, MNRAS, 272, 737

Sanders, D. B., Phinney, E. S., Neugebauer, G., Soifer, B. T., \& Matthews, K. 1989, ApJ, 347, 29

Schneider, G., \& Stobie, E. 2002, in ASP Conf. Ser. 281, Astronomical Data Analysis Software and Systems XI, ed. D. A. Bohlender, D. Durand, \& T. H. Handley (San Francisco: ASP), 382

Shemmer, O., Brandt, W. N., Vignali, C., Schneider, D. P., Fan, X., Richards, G. T., \& Strauss, M. A. 2005, ApJ, 630, 729

Shi, Y., et al. 2005, ApJ, 629, 88

Storrie-Lombardi, L. J., Irwin, M. J., McMahon, R. G., \& Hook, I. M. 2001, MNRAS, 322, 933

Strateva, I. V., Brandt, W. N., Schneider, D. P., Vanden Berk, D. G., \& Vignali, C. 2005, AJ, 130, 387

Tran, H. D., Cohen, M. H., \& Villar-Martin, M. 2000, AJ, 120, 562

Urry, M., \& Padovani, P. 2000, PASP, 112, 1516

Werner, M. W., et al. 2004, ApJS, 154, 1

Willott, C. J., McLure, R. J., \& Jarvis, M. J. 2003, ApJ, 587, L15

Wilkes, B. J., et al. 2000, in ISO Survey of a Dusty Universe, ed. D. Lemke, M. Stickel, \& K. Wilke (Lecture Notes in Physics 548; Berlin: Springer), 177

Young, S., Hough, J. H., Efstathiou, A., Wills, B. J., Bailey, J. A., Ward, M. J., \& Axon, D. J. 1996, MNRAS, 281, 1206

Zakamska, N. L., Strauss, M. A., Heckman, T. M., Ivezić, Ž., \& Krolik, J. H. 2004, AJ, 128, 1002

Zakamska, N. L., et al. 2003, AJ, 126, 2125 $2005, \mathrm{AJ}, 129,1212$ 